\title{
Loop mixing of the opposite parity fermion fields and its manifestation in $\pi N$ scattering
}

\author{
Vladimir Lomov* \\ Institute for System Dynamics and Control Theory of SB RAS, Irkutsk \\ E-mail: v.p.lomov@gmail.com
}

Kaloshin A.E.

Irkutsk State University, Irkutsk

E-mail: shin7@rambler.ru

\section{Kobeleva E.A.}

Irkutsk State University, Irkutsk

E-mail: elenyich@mail.ru

We develop a variant of $K$-matrix, which includes the effect of opposite parity fermions (OPF) mixing, and apply it for description of $\pi N$ partial waves $S_{11}$ and $P_{11}$. OPF-mixing leads to appearance of negative energy poles in $K$-matrix and restoration of MacDowell symmetry, relating two partial waves. Joint analysis of PWA results for $S_{11}$ and $P_{11}$ confirms significance of this effect.

PACS: $13.75 . \mathrm{Gx}, 11.80 . \mathrm{Et}$

XXII International Baldin Seminar on High Energy Physics Problems,

15-20 September 2014

JINR, Dubna, Russia

\footnotetext{
* Speaker.
} 


\section{Introduction}

For fermions there exists a non-standard mixing, when fermion fields with opposite parities are mixing at loop level while parity is conserved in vertex (shortly OPF-mixing):

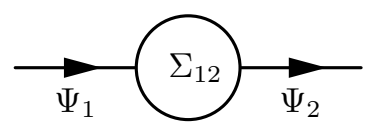

It is possible because fermion and antifermion have different parities. This effect was investigated in detail in Ref. [1] and was applied to $\pi N$ scattering, where it leads to relation between two partial waves. In Ref. [1], the simplest physical example of manifestation of this effect was found: the partial waves $P_{13}$ and $D_{13}$, where baryons $J=3 / 2^{ \pm}$are produced. The OPF-mixing effect is identified in the partial wave $P_{13}$ as rather specific interference of resonance with background generated by resonance state in $D_{13}$ wave. The above-mentioned relation between partial waves mainly influences on a wave with lower orbital momentum and it is used as additional source of information about structure of wave with higher $l$.

Another physical example, where OPF-mixing may be essential, is related to the partial waves $S_{11}$ and $P_{11}$, where resonances $J^{P}=1 / 2^{ \pm}, I=1 / 2$ are produced. Most interesting object here is the Roper resonance $N(1440)$, which has some unusual properties and problems with quarkmodels identification, see, e.g. Refs. [2, 3, 4, 5, 6, 7, 8, 9, 10]. However, in presence of several resonance states the approach of Ref. [1], that uses a matrix propagator, becomes too cumbersome. Alternatively, for description of OPF-mixing, one can use the $K$-matrix approach, which works for any number of states and channels.

Here we present the $K$-matrix approach for $\pi N$ partial amplitudes with accounting of the OPFmixing effect and apply it for description of $S_{11}$ and $P_{11}$ partial waves. Most serious changing as compared with its standard form is the appearance of negative energy poles in $K$-matrix. If, besides, we use QFT to calculate tree amplitudes (i.e. $K$-matrix), starting from effective Lagrangians, we obtain the partial amplitudes $\pi N \rightarrow \pi N$ satisfying the MacDowell symmetry condition:

$$
f_{l,+}(W)=-f_{l+1,-}(-W),
$$

which was obtained in Ref. [11] from general analytic properties of amplitudes.

We use the obtained $K$-matrix to describe results of partial wave analysis for $S_{11}$ and $P_{11}$ amplitudes. The main purpose is to see the manifestation of OPF-mixing and it naturally leads to joint fitting of these two waves.

\section{OPF-mixing and $K$-matrix}

We need to discuss the effect of OPF-mixing in amplitudes of $\pi N$ scattering and its implementation in framework of $K$-matrix description. For a first step one may restrict oneself by a simplified case: two resonance states and two channels.

Effective Lagrangians $\pi N N^{\prime}$ without derivatives and conserving the parity:

$$
\begin{aligned}
& \mathscr{L}_{\text {int }}=g_{1} \bar{N}_{1}(x) N(x) \phi(x)+\text { h.c. }, \quad \text { for } J^{P}\left(N_{1}\right)=1 / 2^{-}, \\
& \mathscr{L}_{\text {int }}=\imath g_{2} \bar{N}_{2}(x) \gamma^{5} N(x) \phi(x)+\text { h.c., } \quad \text { for } J^{P}\left(N_{2}\right)=1 / 2^{+} \text {. }
\end{aligned}
$$


Let us consider two baryon states of opposite parities with masses $m_{1}\left(J^{P}=1 / 2^{-}\right), m_{2}\left(J^{P}=\right.$ $1 / 2^{+}$) and two intermediate states $\pi N, \eta N$. Using the effective Lagrangians we can calculate contributions of states $N_{1}, N_{2}$ to partial waves at tree level:

$s$-wave amplitudes:

$$
\begin{aligned}
& f_{s,+}^{\text {tree }}(\pi N \rightarrow \pi N)=-\frac{\left(E_{N}^{(\pi)}+m_{N}\right)}{8 \pi W}\left(\frac{g_{1, \pi}^{2}}{W-m_{1}}+\frac{g_{2, \pi}^{2}}{W+m_{2}}\right), \\
& f_{s,+}^{\text {tree }}(\pi N \rightarrow \eta N)=-\frac{\sqrt{\left(E_{N}^{(\pi)}+m_{N}\right)\left(E_{N}^{(\eta)}+m_{N}\right)}}{8 \pi W}\left(\frac{g_{1, \pi} g_{1, \eta}}{W-m_{1}}+\frac{g_{2, \pi} g_{2, \eta}}{W+m_{2}}\right), \\
& f_{s,+}^{\text {tree }}(\eta N \rightarrow \eta N)=-\frac{\left(E_{N}^{(\eta)}+m_{N}\right)}{8 \pi W}\left(\frac{g_{1, \eta}^{2}}{W-m_{1}}+\frac{g_{2, \eta}^{2}}{W+m_{2}}\right)
\end{aligned}
$$

and $p$-wave amplitudes:

$$
\begin{aligned}
& f_{p,-}^{\text {tree }}(\pi N \rightarrow \pi N)=\frac{\left(E_{N}^{(\pi)}-m_{N}\right)}{8 \pi W}\left(\frac{g_{1, \pi}^{2}}{-W-m_{1}}+\frac{g_{2, \pi}^{2}}{-W+m_{2}}\right) \\
& f_{p,-}^{\text {tree }}(\pi N \rightarrow \eta N)=\frac{\sqrt{\left(E_{N}^{(\pi)}-m_{N}\right)\left(E_{N}^{(\eta)}-m_{N}\right)}}{8 \pi W}\left(\frac{g_{1, \pi} g_{1, \eta}}{-W-m_{1}}+\frac{g_{2, \pi} g_{2, \eta}}{-W+m_{2}}\right), \\
& f_{p,-}^{\text {tree }}(\eta N \rightarrow \eta N)=\frac{\left(E_{N}^{(\eta)}-m_{N}\right)}{8 \pi W}\left(\frac{g_{1, \eta}^{2}}{-W-m_{1}}+\frac{g_{2, \eta}^{2}}{-W+m_{2}}\right) .
\end{aligned}
$$

Here $W=\sqrt{s}$ is the total CMS energy and $E_{N}^{(\pi)}\left(E_{N}^{(\eta)}\right)$ is nucleon CMS energy of system $\pi N$ $(\eta N)$

$$
E_{N}^{(\pi)}=\frac{W^{2}+m_{N}^{2}-m_{\pi}^{2}}{2 W}
$$

Short notations for coupling constants, e.g. $g_{1, \pi}=g_{N_{1} \pi N}$.

The tree amplitudes (2.3)-(2.4) contain poles with both positive and negative energy, originated from propagators of $N_{1}$ and $N_{2}$ fields of opposite parities. Accounting the loop transitions results in dressing of states and also in mixing of these two fields.

Note that $W \rightarrow-W$ replacement gives

$$
E_{N}^{(\pi)}+m_{N} \rightarrow-\left(E_{N}^{(\pi)}-m_{N}\right),
$$

so tree amplitudes (2.3)-(2.4) exhibit the MacDowell symmetry property [11]

$$
f_{p,-}(W)=-f_{s,+}(-W) .
$$

In $K$-matrix representation for partial amplitudes

$$
f=K(1-\imath P K)^{-1},
$$

diagonal matrix $\imath P$, constructed from CMS momenta, originates from imaginary part of a loop. Therefore, $K$-matrix here is simply a matrix of tree amplitudes that should be identified with amplitudes (2.3), (2.4). 
As a result we come to representation of partial amplitudes for $s$ - and $p$-waves

$$
f_{s}(W)=K_{s}(W)\left(1-\imath P K_{s}(W)\right)^{-1}, \quad f_{p}(W)=K_{p}(W)\left(1-\imath P K_{p}(W)\right)^{-1},
$$

where the matrices $K_{s}, K_{p}$ (i.e. tree amplitudes (2.3), (2.4)), may be written in factorized form

$$
K_{s}=-\frac{1}{8 \pi} \rho_{s} \hat{K}_{s} \rho_{s}, \quad K_{p}=\frac{1}{8 \pi} \rho_{p} \hat{K}_{p} \rho_{p} .
$$

Here $\rho_{s}, \rho_{p}$ are

$$
\begin{aligned}
& \rho_{s}(W)=\left(\begin{array}{cc}
\sqrt{\frac{E_{N}^{(\pi)}+m_{N}}{W}}, & 0 \\
0, & \sqrt{\frac{E_{N}^{(\eta)}+m_{N}}{W}}
\end{array}\right), \\
& \rho_{p}(W)=\left(\begin{array}{cc}
\sqrt{\frac{E_{N}^{(\pi)}-m_{N}}{W}}, & 0 \\
0, & \sqrt{\frac{E_{N}^{(\eta)}-m_{N}}{W}}
\end{array}\right),
\end{aligned}
$$

and matrix $P$ consists of CMS momenta as analytic functions of $W$. In this case "primitive" $K$ matrices contain poles with both positive and negative energies

$$
\begin{aligned}
& \hat{K}_{s}(W)=\left(\begin{array}{l}
\frac{g_{1, \pi}^{2}}{W-m_{1}}+\frac{g_{2, \pi}^{2}}{W+m_{2}}, \frac{g_{1, \pi} g_{2, \eta}}{W-m_{1}}+\frac{g_{2, \pi} g_{2, \eta}}{W+m_{2}} \\
\frac{g_{1, \pi} g_{2, \eta}}{W-m_{1}}+\frac{g_{2, \pi} g_{2, \eta}}{W+m_{2}}, \frac{g_{1, \eta}^{2}}{W-m_{1}}+\frac{g_{2, \eta}^{2}}{W+m_{2}}
\end{array}\right), \\
& \hat{K}_{p}(W)=\hat{K}_{s}(-W)=\left(\begin{array}{l}
\frac{g_{1, \pi}^{2}}{-W-m_{1}}+\frac{g_{2, \pi}^{2}}{-W+m_{2}}, \frac{g_{1, \pi} g_{2, \eta}}{-W-m_{1}}+\frac{g_{2, \pi} g_{2, \eta}}{-W+m_{2}} \\
\frac{g_{1, \pi} g_{2, \eta}}{-W-m_{1}}+\frac{g_{2, \pi} g_{2, \eta}}{-W+m_{2}}, \frac{g_{1, \eta}^{2}}{-W-m_{1}}+\frac{g_{2, \eta}^{2}}{-W+m_{2}}
\end{array}\right) .
\end{aligned}
$$

Recall that $m_{1}$ is mass of $J^{P}=1 / 2^{-}$state and $m_{2}$ is mass of $J^{P}=1 / 2^{+}$one. Generalization of this construction for the case of more channels and states is obvious.

Since CMS momenta have the property $P(-W)=-P(W)$, the MacDowell symmetry property (2.7) is extended from tree amplitudes to unitarized $K$-matrix ones (2.9).

From a common sense one can expect that negative energy pole should give a negligible effect in physical energy region. However, this is not the case if corresponding coupling constant is large $\left|g_{2, \pi}\right| \gg\left|g_{1, \pi}\right|$. One can compare decay width of $s$ - and $p$-states

$$
\Gamma\left(N_{1} \rightarrow \pi N\right)=g_{N_{1} \pi N}^{2} \Phi_{s}, \quad \Gamma\left(N_{2} \rightarrow \pi N\right)=g_{N_{2} \pi N}^{2} \Phi_{p},
$$

where $\Phi_{s}, \Phi_{p}$ are corresponding phase volumes. For resonance states not far from threshold, with masses, e.g. 1.5-1.7 GeV, phase volumes differ greatly, $\Phi_{s} \gg \Phi_{p}$. If both resonances have typical hadronic width $\Gamma \sim 100 \mathrm{MeV}$, then coupling constants differ dramatically too, $\left|g_{N_{2} \pi_{N}}\right| \gg\left|g_{N_{1} \pi N}\right|$. 
Above we use the simplest effective Lagrangians (2.1)-(2.2) to derive tree amplitudes. However, it is well-known, that spontaneous breaking of chiral symmetry requires pion field to appear in Lagrangian only through derivatives

$$
\mathscr{L}_{\text {int }}=f_{2} \bar{N}_{2}(x) \gamma^{5} \gamma^{\mu} N(x) \partial_{\mu} \phi(x)+\text { h.c., } J^{P}=1 / 2^{+}, f_{2}=\frac{g_{2}}{m_{2}+m_{N}} .
$$

It is not difficult to understand how inclusion of derivative changes tree amplitudes and, hence $K$-matrix. Pole contribution $\pi\left(k_{1}\right) N\left(p_{1}\right) \rightarrow N_{2}(p) \rightarrow \pi\left(k_{2}\right) N\left(p_{2}\right)$ in that case takes the form:

$$
T=f_{2}^{2} \bar{u}\left(p_{2}\right) \gamma^{5} \hat{k}_{2} \frac{1}{\hat{p}-M} \gamma^{5} \hat{k}_{1} u\left(p_{1}\right) .
$$

With the use of equations of motion, we see that inclusion of derivative at vertex leads to the following modification of resonance contribution

$$
g_{2}^{2} \frac{1}{\hat{p}-M} \rightarrow f_{2}^{2}\left(\hat{p}+m_{N}\right) \frac{1}{\hat{p}-M}\left(\hat{p}+m_{N}\right) .
$$

Separation of the positive and negative energy poles is performed with the off-shell projector operators $\Lambda^{ \pm}=1 / 2(1 \pm \hat{p} / W)$

$$
f_{2}^{2}\left(\hat{p}+m_{N}\right) \frac{1}{\hat{p}-m_{N}}\left(\hat{p}+m_{N}\right)=\Lambda^{+} \frac{f_{2}^{2}\left(W+m_{N}\right)^{2}}{W-M}+\Lambda^{-} \frac{f_{2}^{2}\left(W-m_{N}\right)^{2}}{-W-M},
$$

where the first term gives contribution to $p$-wave and second one to $s$-wave. Modification of the pole contributions in "primitive" $K$-matrices (2.13)-(2.14) is evident

$$
\begin{array}{ll}
g_{2}^{2} \rightarrow f_{2}^{2}\left(W-m_{N}\right)^{2}, & \text { for } s \text {-wave, } \\
g_{2}^{2} \rightarrow f_{2}^{2}\left(W+m_{N}\right)^{2}, & \text { for } p \text {-wave. }
\end{array}
$$

One can expect that the inclusion of derivatives most strongly affects on threshold properties of $s$-wave due to dumping factor $\left(W-m_{N}\right)^{2}$.

\section{Partial amplitudes $P_{11}$ and $S_{11}$ of $\pi N$ scattering}

First of all, let us try to describe $S_{11}$ and $P_{11}$ waves separately. $p$-wave is described rather well by our formulas with derivative in vertex (2.20)- (2.21), see Fig. 1 where solid lines represent our amplitudes (2.9)-(2.14) in the presence of derivative in vertex (2.20)- (2.21). In this case the $s$-wave states are missing in amplitudes, the $p$-wave $K$-matrix has two positive energy poles.

Quality of description is defined by:

$$
\chi^{2} / \mathrm{DOF}=273 / 95
$$

The use of vertices without derivative leads to impairment of quality of description: $\chi^{2}>350$, again we need two poles with close masses.

Both variants give a negative background contribution to $S_{11}$ wave, comparable in magnitude with other contributions, as it seen on Fig. 2. This figure shows background contribution to $s$ wave generated by $p$-wave states, i.e. in this case $K$-matrix for $s$-wave (2.13) has only negative 

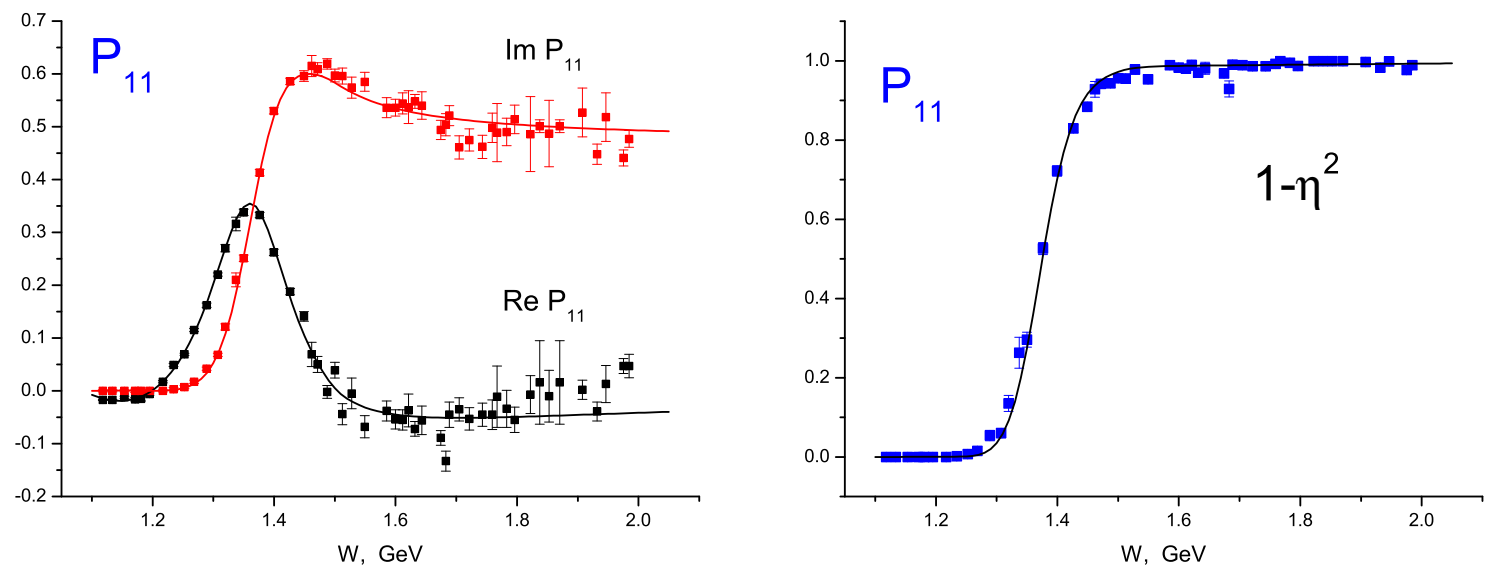

Figure 1: The results of fitting of $P_{11}$-wave of $\pi N$ scattering. Dots show results of PWA [12], solid lines represent our amplitudes in the presence of derivative in vertex. $K$-matrix has only $p$-wave states. On the right side: $p$-wave inelasticity [12], the curve corresponds to lines on the left side. Partial wave normalization corresponds to Ref. [12]: $\operatorname{Im} f=|f|^{2}+\left(1-\eta^{2}\right) / 4$.

energy poles. Variant without derivative in vertex gives a larger background contribution, rapidly changing near thresholds. It seems that description of $P_{11}$ partial wave without derivative in vertices contradicts to data on $S_{11}$. On Fig. 2 some typical curves are shown, there exist different variants with sharp behavior near thresholds. The presence of derivative in a vertex suppresses the threshold region in background contribution due to factor $\left(W-m_{N}\right)^{2}$, but in resonance region this is rather large contribution, see Fig. 2.

Attempt to describe $S_{11}$ without background has no success: it doesn't allow to reach even qualitative agreement with PWA.

As a next step, let us add the background contribution, arising from $p$-wave states (solid lines on Fig. 1) with fixed parameters of $p$-wave.

One can see from Fig. 3 that quality of description is unsatisfactory in this case but doublepeak behavior is arisen in partial wave for the first time. It means that to describe $S_{11}$ wave a background contribution is necessary and its value is close to solid line curves at Fig. 1

\section{Joint fit of $S_{11}$ and $P_{11}$}

Let's perform the joint analysis of $S_{11}$ and $P_{11}$ amplitudes, when resonance states in one wave generate background in other and vice versa. In this case $K$-matrices (2.13)-(2.14) have poles with both positive and negative energies: we use two $s$-wave and two $p$-wave poles. This leads to noticeable improvement of description, as can be seen from Fig. 4 ; in this case $\chi^{2} / \mathrm{DOF}=850 / 190$.

At last, background can be generated not only by negative energy poles but by other terms. We accounted it by adding to elastic amplitudes $\pi N \rightarrow \pi N$ a smooth contributions of the form:

$$
\hat{K}_{s}^{B}=A+B\left(W-m_{N}\right)^{2}, \quad \hat{K}_{p}^{B}=A+B\left(W+m_{N}\right)^{2},
$$




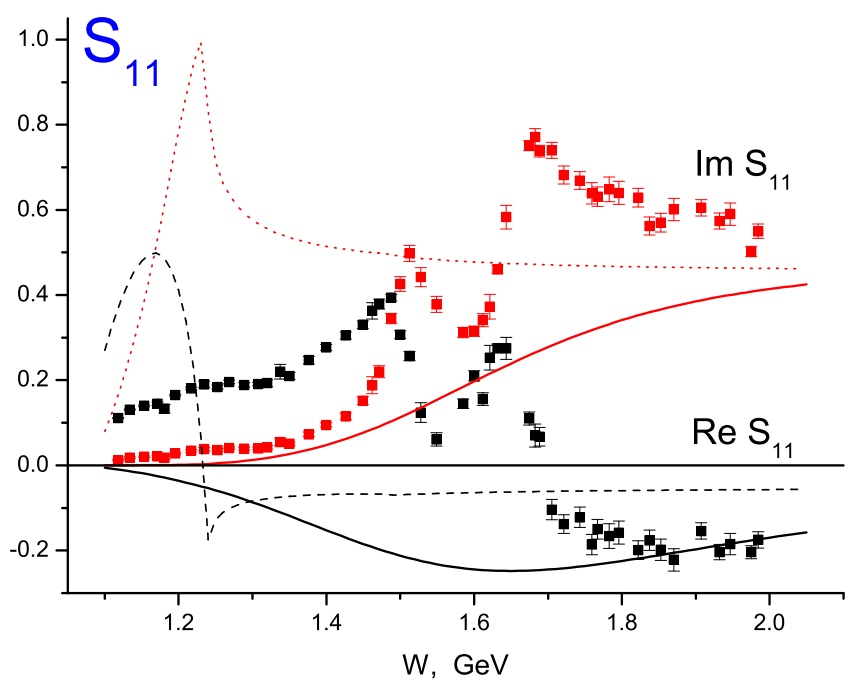

Figure 2: Background contribution to $s$-wave, generated by $p$-wave states. Solid lines represent variant with derivative in vertex (corresponding to curves on Fig. 1), dashed lines - variant without derivative in vertex.

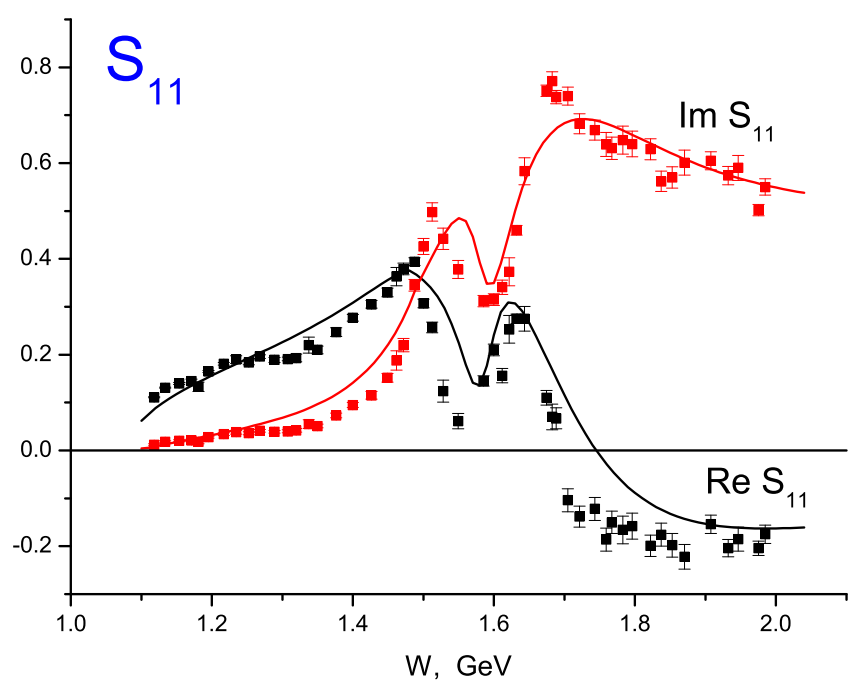

Figure 3: Results of $s$-wave fitting with fixed parameters for $p$-wave states. Parameters of $p$-wave correspond to curves on Fig. 1, s-wave contains two states with $K$-matrix masses 1.55 and $1.75 \mathrm{GeV}$.

which do not violate the MacDowell symmetry property. Note that we have quite good description $\chi^{2} / \mathrm{DOF}=584 / 187$ and background contribution in $S_{11}$ is close to simplest variant of Fig. 2. 

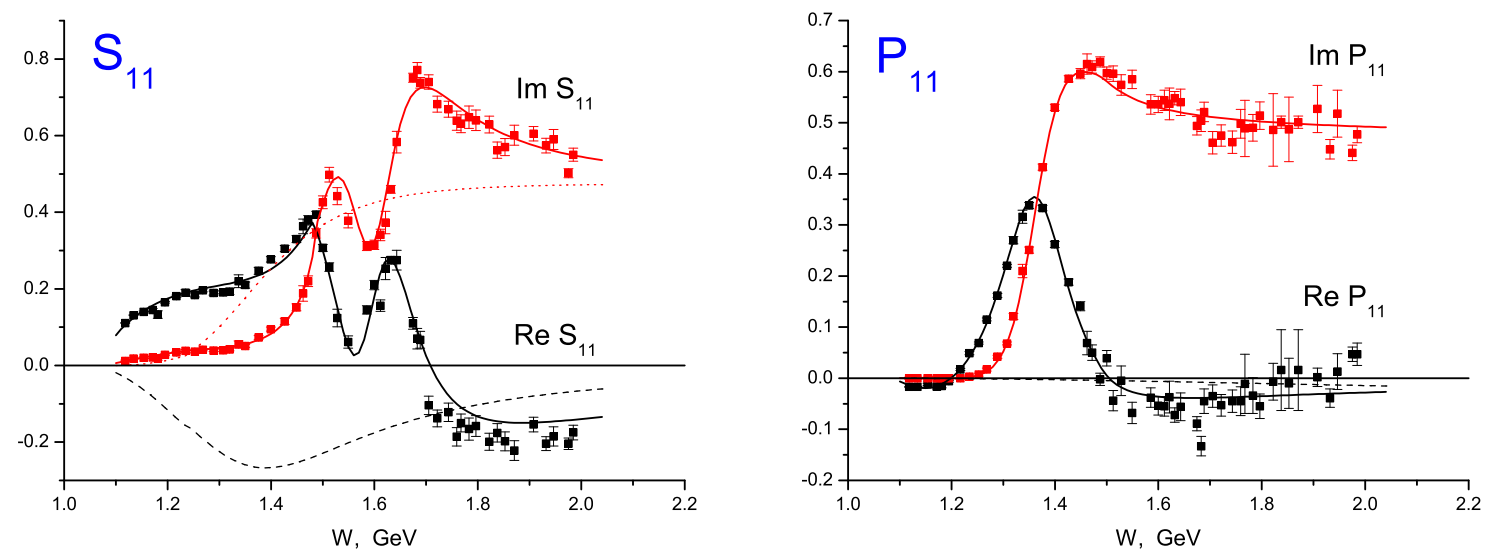

Figure 4: Result of joint fitting of $S_{11}$ and $P_{11}$-waves of $\pi N$ scattering. Dashed lines show real and imaginary parts of (unitarized) background contribution.
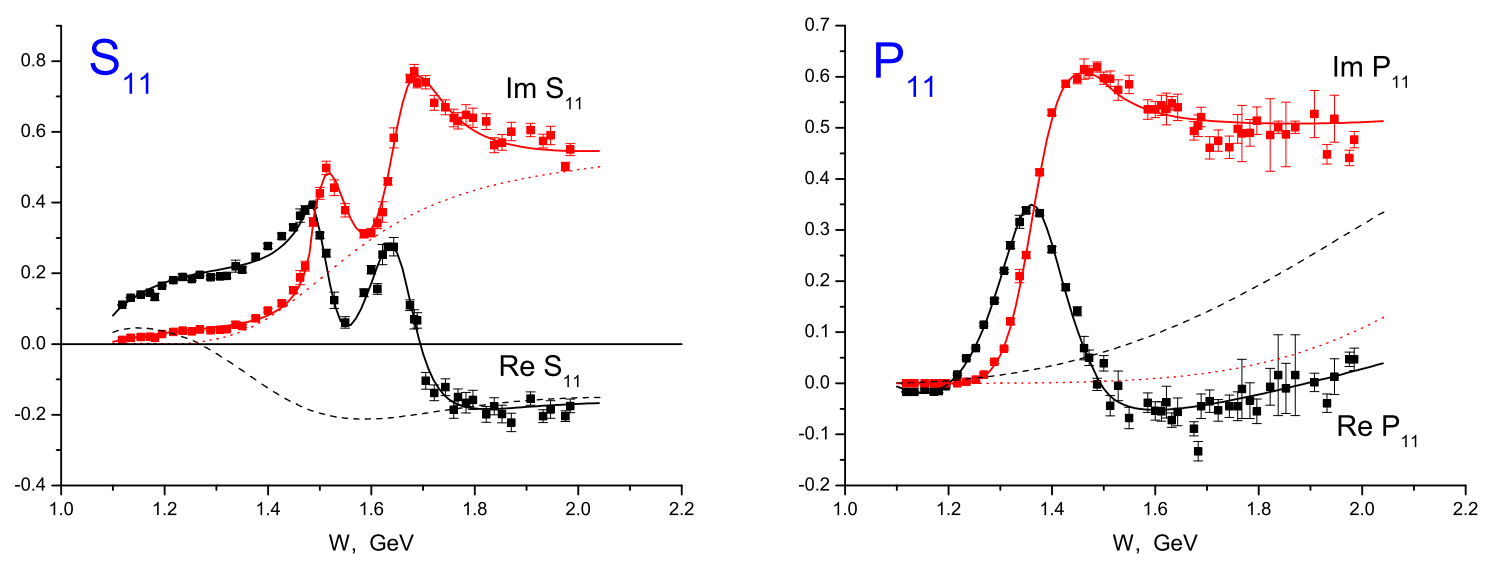

Figure 5: Result of joint fitting of $S_{11}$ and $P_{11}$ waves of $\pi N$ scattering.

\section{Poles in complex plane}

In Table 1 we present the pole masses and widths obtained by continuation of our amplitudes to complex $W$ plane. As a whole, we see that our values for $m_{p}, \Gamma_{p}$ are rather close to previously obtained. The only hint for disagreement is appearance at some sheets of a stable pole $1 / 2^{+}$with $m_{p} \approx 1500 \mathrm{MeV}$ instead of generally accepted mass $m_{p} \approx 1365 \mathrm{MeV}$.

\section{Conclusions}

In this report we investigated the manifestation of OPF-mixing in $\pi N$ partial waves $S_{11}$ and $P_{11}$, where baryons $1 / 2^{ \pm}, I=1 / 2$ are produced. We found that the effect of mixing of fermion fields with opposite parity can be readily realized in the framework of $K$-matrix approach. It allows to 


\begin{tabular}{|c|c|c|}
\hline $\begin{array}{c}\text { Partial wave, } \\
\text { PDG values }\end{array}$ & This work & Some other works \\
\hline $\begin{array}{c}S_{11}, 1 / 2^{-} \\
\mathrm{N}(1535)(1510,70)\end{array}$ & $(1507,87)$ & $(1502,95),(1648,80)[12]$ \\
$\mathrm{N}(1650)(1655,165)$ & $(1659,149)$ & $(1519,129),(1669,136)[13]$ \\
\hline$P_{11}, 1 / 2^{+}$ & $(1365,194)$ & $(1359,162)[12]$ \\
$\mathrm{N}(1440)(1365,190)$ & $(1500,160)$ & $(1385,164)[14]$ \\
& & $(1387,147)[13]$ \\
\hline
\end{tabular}

Table 1: Pole masses and widths $\left(M_{R}, \Gamma_{R}\right)$ extracted from poles position in the complex plane $W: W_{0}=$ $M_{R}-\imath \Gamma_{R} / 2$.

have simple expressions for amplitudes in the case of any resonance states and reaction channels. Note that $s$ - and $p$-wave $K$-matrices, (2.13)-(2.14), have poles with both positive and negative energies and are related with each other by $\hat{K}_{p}(W)=\hat{K}_{s}(-W)$.

The so-constructed partial waves possess the well-known MacDowell symmetry that connects two partial waves under substitution $W \rightarrow-W$. Up to now, this symmetry did not play any role in data analysis since it connects physical and unphysical regions. However, taking OPF-mixing into account, MacDowell symmetry leads to physical consequences: resonance in one partial wave gives rise to background contribution in another and vice versa. This connection between two waves, as in case of $3 / 2^{ \pm}$resonances,[1] works mainly in one direction: it generates large negative background in a wave with lower orbital momentum. So we come to idea of joint analysis of two partial waves and it allows to get an additional information about dynamics in higher $l$ wave. Such an example can be seen at Fig. 2, where two variants of background in $S_{11}$ are depicted.

Our main purpose here was to see the effects of OPF-mixing in the amplitudes $S_{11}, P_{11}$ and to estimate their value. So, following Ref. [15], we have used simplified three-channel formalism in which $\sigma N$ is some quasi-channel, imitating different $\pi \pi N$ intermediate states. In spite of the rough approach we obtained rather good description of $S_{11}$ and $P_{11}$ waves, comparable well with more comprehensive analyses $[16,17,18,19]$ with number of channels up to 6 . We suppose that OPF-mixing (or MacDowell symmetry) can be taken into account not only in $K$-matrix formalism but in framework of more detailed dynamical multi-channel approach.

Note that obtained pole positions not always coincide with the results of previous analyses. For example, for $N(1440)$ state we found on most sheets a very stable pole with $\operatorname{Re} W \approx 1500 \mathrm{MeV}$ instead of "standard" value $\approx 1360 \mathrm{MeV}$, see Table 1 . After various verifications we suppose that this is result of crudity of used approximation (effective $\sigma N$ channel). But it is possible that there exists some dependency on details of description and it needs more close investigation.

Summarizing, we found out that effect of a loop OPF-mixing is seen in PWA results as a connection between partial waves $S_{11}$ and $P_{11}$. We assume that this connection may be of interest as possibility to obtain additional information about $P_{11}$ wave and baryons $1 / 2^{+}$. 


\section{References}

[1] A.E. Kaloshin, E.A. Kobeleva, and V.P. Lomov, Mixing of fermion fields of opposite parities and baryon resonances, Int.J.Mod.Phys. A26 2307 (2011).

[2] O. Krehl, C. Hanhart, S. Krewald, and J. Speth, What is the structure of the Roper resonance?, Phys.Rev. C62 025207 (2000).

[3] M. Batinic, I. Slaus, A. Svarc, and B.M.K. Nefkens, $\pi N \rightarrow \eta N$ and $\eta N \rightarrow \eta N$ partial wave $T$ matrices in a coupled, three channel model, Phys.Rev. C51 2310 (1995).

[4] L.Ya. Glozman and D.O. Riska, The Spectrum of the nucleons and the strange hyperons and chiral dynamics, Phys.Rept. 268 (1996).

[5] S. Capstick and W. Roberts, Quark models of baryon masses and decays, Prog.Part.Nucl.Phys. 45 $241(2000)$.

[6] N. Mathur, Y. Chen, S.J. Dong, T. Draper, I. Horvath, et al, Roper resonance and $S_{11}(1535)$ from lattice QCD, Phys.Lett. B605 137 (2005).

[7] M. Dillig and M. Schott, Mesonic content of the nucleon and the Roper resonance, Phys.Rev. C75 067001 (2007).

[8] A.V. Sarantsev, M. Fuchs, M. Kotulla, U. Thoma, J. Ahrens, et al, New results on the Roper resonance and the $P(11)$ partial wave, Phys.Lett. B659 94 (2008).

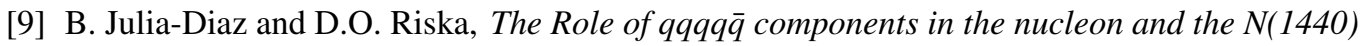
resonance, Nucl.Phys. A780 175 (2006).

[10] C.D. Roberts, Hadron Properties and Dyson-Schwinger Equations, Prog.Part.Nucl.Phys. 6150 (2008).

[11] S.W. MacDowell, Analytic properties of partial amplitudes in meson-nucleon scattering, Phys. Rev. 116774 (1959).

[12] R.A. Arndt, W.J. Briscoe, I.I. Strakovsky, and R.L. Workman, Extended partial-wave analysis of $\pi N$ scattering data, Phys.Rev. C74 045205 (2006).

[13] M. Doring, C. Hanhart, F. Huang, S. Krewald, and U.-G. Meissner, Analytic properties of the scattering amplitude and resonances parameters in a meson exchange model, Nucl.Phys. A829 170 (2009).

[14] G. Hohler, What can be learned from elastic electron-nucleon scattering experiments?, $\pi N$ Newslett. 108 (1993).

[15] S. Ceci, J. Stahov, A. Svarc, S. Watson, and B. Zauner, Resolution of the multichannel anomaly in the extraction of S-matrix resonance-pole parameters, Phys.Rev. D77 116007 (2008).

[16] S.X. Nakamura, Extraction of $P_{11}$ resonance from $\pi N$ data and its stability, AIP Conf.Proc. 1374505 (2011).

[17] H. Kamano, S.X. Nakamura, T.-S.H. Lee, and T. Sato, Extraction of $P_{11}$ resonances from $\pi N$ data, Phys.Rev. C81 065207 (2010).

[18] B. Golli and S. Sirca, Roper resonances in chiral quark models, Eur.Phys.J. A38 271 (2008).

[19] M.W. Paris and R.L. Workman, Toward a unified description of hadro- and photoproduction: S-wave $\pi$ - and $\eta$-photoproduction amplitudes, Phys.Rev. C82 035202 (2010). 